\title{
6 Quantum computing and classical politics
}

\section{The ambiguity of advantage in signals intelligence}

\author{
Jon R. Lindsay
}

The discovery of quantum mechanics in the early 20th century transformed our understanding of subatomic reality. A "second quantum revolution" (Dowling and Milburn 2003) in the 21 st century is poised to transform our ability to manipulate subatomic reality to process information. It is possible to perform calculations with quantum bits (qubits) that are practically infeasible with classical (digital) bits. Potential applications of quantum computing may improve remote sensing, data processing, and secure networking, all of which might affect global security (Biercuk and Fontaine 2017; National Academies of Sciences, Engineering, and Medicine 2019). These give rise to excited claims about how the "impact of quantum on our national defense will be tremendous" (Hurd 2017) or that "whoever gets this technology first will also be able to cripple traditional defenses and power grids and manipulate the global economy" (Nikias 2018). China's early progress in quantum communication technology has further energized the global race to gain a quantum advantage (Owen and Gorwa 2016; Kania 2018).

How will the second quantum revolution affect global cyber security in particular? A stylized debate has unfolded in the field of international relations (IR) about the relationship between information and communication technology (ICT) and conflict dynamics. One side argues that the nature of cyberspace is strategically destabilizing because it empowers the offense, weakens the defense, or undermines deterrence (Rattray 2001; Lynn III 2010; Kello 2013; Deibert 2013; Buchanan 2017; Schneider 2019). The other side argues that the sociopolitical context of cyber security mitigates the potency of offensive cyber operations and reinforces established power relations (Libicki 2007; Rid 2012; Liff 2012; Gartzke 2013; Lindsay 2014; Valeriano and Maness 2015; Slayton 2017; Kreps and Schneider 2019). In between these extremes scholars highlight variable conditions that influence the difficulty of attribution, offensive advantage, or strategic stability (Rid and Buchanan 2015; Lindsay 2015; Brantly 2016; Smeets 2017; Borghard and Lonergan 2017). Others highlight problems of threat inflation and motivated bias that distort the rhetoric of cyber war (Dunn Cavelty 2008; Lawson 2013).

Because ICT is so vital for everything in the modern world, it is reasonable to expect a new generation of ICT - from bit to qubit - to be particularly consequential. If we believe that technology determines politics, then we might expect 
a fundamental change in the underlying infrastructure of cyberspace to have dramatic consequences. If, however, we believe that politics tends to determine technology, then the consequences of the quantum information revolution might not be so profound after all. A more reciprocal or endogenous interaction between them, moreover, could have even more ambiguous implications.

This chapter puts quantum information technology into political context. I focus on the applications for code making (cryptography) and code breaking (cryptanalysis) because they are particularly dramatic. I start with a brief summary of quantum threat narratives. I then explain why all cryptologic phenomena are fundamentally political, no matter the vintage of technology that implements them. Secret communication necessarily depends on cooperation between communicators seeking to exclude another group of competitors. Yet secret eavesdropping also depends on cooperation, since the interloper must adopt the same communicative protocols to gain and maintain covert access. In any practical context, moreover, both sides of the cryptologic contest must deal with organizational and strategic challenges that can either bolster or degrade the security of technical cryptosystems. Scientific innovation in quantum technology will not change, and in many ways will exacerbate, the social interactions that make cryptology a complex and ambiguous practice. I conclude with a few brief remarks on how social scientists can recover quantum technology from the technologists.

\section{Quantum threat narratives}

Quantum computing leverages "spooky" quantum phenomena like superposition (the ability for a qubit to be in two states at once), entanglement (the ability for multiple qubits to influence each other from a distance), and indeterminacy (the tendency for measurement to affect the state of reality). Richard Feynman first suggested the idea several decades ago as a promising way to model physical chemistry (Feynman 1982). Experimental progress was slow to catch up, but that is changing quickly. In September 2019, a working quantum computer designed by Google and known as Sycamore achieved a major performance milestone known as "quantum supremacy". Sycamore entangled 53 qubits and ran a quantum algorithm in mere minutes that would take the world's fastest supercomputer thousands of years to complete (Arute et al. 2019). ${ }^{1}$

Quantum computing has important implications for cryptography, which is vital for cyber security, which in turn has become a pressing concern for governments, commercial firms, and civil society around the world. Today, nearly all secure digital communication relies on a small number of cryptographic protocols such as RSA (Rivest-Shamir-Adleman). RSA is the standard used for most implementations of public key infrastructure (PKI), which links real people and organizations to the cryptographic keys they use for secure communications and digital signatures. The categorical compromise of PKI, which would enable a hacker to break encryption, steal data, forge signatures, and install arbitrary code, would be a disaster for global trade, national security, and civil society 
(Mulholland, Mosca, and Braun 2017). The security of PKI today is predicated on the computational difficulty of solving (but easily verifying) certain mathematical problems such as factoring large prime numbers. A typical digital computer would need six quadrillion years to crack 2048-bit RSA (DigiCert 2018), but a working quantum computer with 20 million qubits might be able to perform the same feat in eight hours (Gidney and Ekera 2019).

There are major engineering challenges to overcome before it is possible to build such a machine. Quantum error correction is particularly daunting, so a working large-scale universal quantum computer may be decades away. Nevertheless, the cryptanalytic threat posed by quantum computing is plausible enough that the cryptographic community has already moved to develop "quantum safe", "quantum-resistant", or "post-quantum" protocols (Lindsay 2020b). These are mathematical functions that are difficult for both classical and quantum computers, which only provide performance gains if a suitable quantum algorithm can be found. There are several promising classical protocols that are already under review by technical standards setting agencies like the US National Institute of Standards and Technology (Chen et al. 2016; Bernstein et al. 2017). These will almost certainly be available before a working machine powerful enough to break RSA is fielded, but transitioning to the new protocols in practice will be a long and bumpy journey for industry and government (Buchmann, Lauter, and Mosca 2017).

While quantum computing poses an offensive (cryptanalytic) threat to asymmetric encryption, quantum mechanics can also be harnessed to improve defense (cryptography). Quantum encryption protocols are guaranteed secure by the laws of physics rather than just the computational difficulty of particular mathematical problems (Lo and Lütkenhaus 2007; Brassard 2016). There are many other potential applications of quantum communications, all with formidable engineering challenges, to include radically different memory devices and internet architectures (Simon 2017; Wehner, Elkouss, and Hanson 2018). Yet there are also promising signs of progress in this area. China has already built a prototype quantum communication network between Beijing and Shanghai and conducted experiments in quantum entanglement with a satellite link (Kania and Costello 2018).

Progress in communications security seems like a good development, and in general it is. Yet the potential of unbreakable security also gives rise to a different sort of threat narrative. If quantum cryptanalysis augurs the end of confidentiality or, more breathlessly, the "cryptocalypse", then quantum cryptography raises a reciprocal fear that intelligence agencies will be locked out or "go dark". Western governments unable to penetrate the communications of terrorist or state adversaries could be denied warning of surprise attack. Police agencies, similarly, could lose access to forensic data in criminal investigations. All of these concerns feed fears that China, an emerging leader in quantum technology, may become the first to gain a destabilizing cryptologic advantage over the United States and others (Owen and Gorwa 2016; Kenny 2017; Kania and Costello 2018).

It is important to recognize that quantum communications and quantum computing are two different categories of technology. China is making impressive 
progress in the former but not the latter, where by contrast North America and Europe maintain a strong lead. For the purposes of this chapter, these two technologies have applications on opposite ends of the cryptologic contest between cryptography and cryptanalysis, respectively. I will now set aside the technical mysteries of quantum computing and explore the political context in which any quantum technologies will be employed.

\section{The political essence of cryptology}

Quantum mechanics describes the nature of reality at the microscale, but the people who will use quantum computers live at the macroscale. Indeed, political context is so important for information technology that it almost ceases to be context at all. In particular there are political problems lurking at the very heart all cryptography, quantum or otherwise.

As David Kahn points out in his magisterial history, "Cryptology is, by definition, a social activity" (Kahn 1996: 752). Yet it is a paradoxical social activity that combines communication, which enables all manner of political economic interaction, with secrecy, which impedes communication and defines social barriers. According to Kahn, "secrecy is the antithesis of communication, and communication - as that which makes man a social being - encompasses all aspects of cultural behavior. Cryptography combines these antitheses into a single operation; a wag might define it as "noncommunicating communication"" (Kahn 1996: 753). This stark contradiction is the source of most of the complexities and conundrums in intelligence practice.

Formal theories of communication and cryptography share the same mathematical foundations. It is no coincidence that the author of a seminal paper on information theory (Shannon 1948) also wrote a classified paper for Bell Labs three years earlier on cryptography (Shannon 1945), using nearly identical concepts. Communication and cryptology deal with the same abstract problems of signal and noise. Communicators try to get their signal transmitted through the noise, while cryptographers try to disguise their signals as noise. The goal of the cryptanalyst, on the other hand, is to recover the signal through the noise that the cryptographer creates. Seen from this perspective, cryptanalysts are communicators too. The same communicative protocols used by two notional conspirators, Alice and Bob, can be exploited by Eve the eavesdropper to read their correspondence or inject disinformation. Yet Eve must take pains to keep her cryptanalytic coup hidden to avoid alerting Alice or Bob, who would then take action to lock her out. To paraphrase Kahn, a wag might define cryptanalysis as "communicating noncommunication".

The rival twins of cryptology - cryptography and cryptanalysis - embody a fundamental political paradox. The cryptologic competitors must respect the constraints of a cooperatively produced cryptosystem. They must cooperate to compete. Indeed, this is a general political feature of intelligence and cyber operations, which rely on stealth and duplicity to penetrate and exploit the cultural norms and sociotechnical institutions that enable cooperation within the target organization 
(Gartzke and Lindsay 2015). Alice and Bob must be simultaneously concerned with managing the internal interactions within their common group as well as foiling external interactions with rival groups (Eve and her co-conspirators). This makes cryptology a two-level game (Putnam 1988), much as national leaders are simultaneously paying attention to foreign and domestic rivals, but on a smaller scale with tight constraints on communicative interaction.

To communicate in secret, Alice and Bob must agree in advance to use a cryptosystem - practices and technologies that enable them to pass meaningful signals to each other that will look like meaningless noise to Eve. There are three general strategies. First, Alice and Bob might agree to conceal the existence of the messages. This works only if Eve doesn't know where to look or is physically prevented from access. For example, steganography hides text within an image, spies leave messages in hidden dead drops, and "air gaps" isolate computers from the public internet to preclude digital access. Yet Alice and Bob will not be able to hide all messages, especially if they are broadcast over open radio or computer networks. Second, Alice and Bob might agree on a method to disguise the meaning. Alice and Bob can encode messages by substituting letters and phrases according to some prepared translation scheme like a codebook, but all the effort is lost if the codebook falls into Eve's hands. A foreign language can be an effective code (e.g., the Navajo Code Talkers used by the United States in World War II), so long as the enemy has no native speakers or talented linguists. Finally, Alice and Bob can encipher the message using mathematical algorithms or computing machines that systematically scramble the message. This has the advantage that Alice and Bob can communicate anything secretly even if Eve knows what cryptosystem they are using, so long as Alice and Bob keep their cipher key(s) secret. As Shannon points out, "one must expect his system to be found out eventually through espionage, captured equipment, prisoners, etc." (Shannon 1945: 25). Modern digital encryption both encodes text and images into ones and zeros and enciphers the coded data via cryptographic algorithms (Kahn 1996: xv-xviii; Singh 1999).

If Eve can steal or discover Alice and Bob's key(s), then she can participate in their shared communicative institution, but she must do so surreptitiously. If apparent noise or pseudo-randomness is the cryptographer's friend, then nonrandomness or redundancy is the cryptanalyst's friend. Indeed, redundancy is any communicator's friend since redundancy enables error correction (Shannon 1951), no matter whether the errors are the result of technical noise or cryptographic obfuscation. To recover the signal from the noise created by Alice and Bob, Eve can study the traffic patterns of their encrypted communications to learn something about the enemy organization (Thirsk 2001). She can perform a brute force attack and try every possible key combination. She can perform frequency analysis across a large volume of intercepted cipher-text to find patterns that narrow the search for keys. She can look for redundant enemy communications or cribs that correlate enciphered messages with known or suspected bits of plaintext (e.g., a morning weather broadcast, a mayday call from a sinking ship, or a lazy operator reusing old keys). She can monitor side-channels in the technical 
infrastructure of the cryptosystem. For example, a pseudorandom number-generating algorithm may give off detectable signals as computing hardware. The common theme is the discovery of a subtle signal in the subterfuge of noise.

Perfect secrecy is possible if a perfectly random key the same length as the message is used only once for each message (Shannon 1945). Unfortunately, there are practical challenges in generating and distributing a so-called one-time pad, as the Soviet KGB and others found to their detriment (Warner and Benson 1997). Quantum key distribution, interestingly, makes one-time pads practically feasible. Most practical classical cryptosystems, by contrast, use short keys that can be easily stored or remembered, using efficient encryption and decryption processes that can work in arduous field conditions. Because such practical systems cannot produce truly randomized messages, they aim to "maximize the minimum amount of work the enemy must do to break it", as Shannon points out; unfortunately, "in the history of cryptography there have been many ciphers which were at first thought unbreakable but later disclosed weaknesses of their own" (Shannon 1945: 87). The advent of quantum networks appears to be just such a moment, heralding new and unbreakable encryption.

\section{Cryptology as an organizational contest}

The logic of cryptology as cooperative competition, or communicating noncommunication, is fundamentally political. The implementation of cryptology piles on additional social factors. Kahn observes that "cryptography and cryptanalysis are sometimes called twin or reciprocal sciences, and in function they indeed mirror one another. What one does the other undoes. Their natures, however, differ fundamentally. Cryptography is theoretical and abstract. Cryptanalysis is empirical and concrete" (Kahn 1996: 737). This has important organizational implications. Alice and Bob and Eve all have to build reliable institutional platforms from which to carry out their complementary yet agonistic activities.

Eve must be both methodical and inventive to break in and collect data. She must collect and analyze her targets' communications, develop programs or build computers that can decrypt them, and find needles of relevant intelligence in haystacks of useless chatter. These usually pose difficult collective problems that require some degree of resourcing and bureaucratic process to accomplish. It generally takes a long time for Eve to prepare her attack, but only a short time for Alice and Bob to lock her out if she is discovered. All of Eve's effort may be undone if Alice and Bob discover that they have been compromised. For this reason, cryptanalytic organizations must also rely on cryptography to cover the internal communications and coordination that makes their exploitation possibleoffense plays defense. Figuratively speaking, Eve must encrypt her decryption. Eve must carefully consider the "intelligence gain-loss" trade-offs between acting on intelligence, which may reveal the source to the target, and not acting, so as to keep on collecting in the future. To mitigate this tradeoff, Eve may try to disguise or "sanitize" the source of intelligence, for instance distributing a sensitive signal intercept as if it were a tip from a human agent. 
Cryptanalysis increasingly relies on computers. Machines can efficiently search through the pseudo-noise created by Alice and Bob by calculating faster, and with larger memories, than humans working alone. As Kahn points out, "World War II mechanized cryptography and mathematized cryptanalysis" (Kahn 1996: 612). Bletchley Park built electromechanical machines known as Bombes, designed by Bletchley's star cryptographer Alan Turing, to crack the German Enigma machine, a mechanical cipher device used by German forces in the field. Another machine known as Colossus, designed by Turing's mentor Max Newman and the talented engineer Tommy Flowers, was able to defeat the Lorentz teleprinter cipher used by Nazi high command. The world's first programmable, digital, electronic computer was actually Colossus, not the more famous ENIAC (Copeland 2010). Wartime cryptography thus gave rise to both Shannon's information theory and modern digital computers.

One enduring lesson from the history of Bletchley Park is that while cryptology relies on mathematics, intelligence is fundamentally a contest between human organizations (Ratcliff 2006; Grey 2012).

The best signals intelligence (SIGINT) will be useless if Eve and her cronies cannot gain access to, make sense of, and act on Alice and Bob's data. The best cryptosystem is useless, likewise, if Alice and Bob have poor operations security (OPSEC) practices that inadvertently expose data and keys to Eve. We do not have to wait for quantum cryptography to understand why strong cryptosystems might not provide reliable cyber security. Unbreakable asymmetric cryptography like RSA has been available for decades, yet overly complex implementations of PKI and poor "cyber hygiene" among computer users are, nevertheless, responsible for the epidemic of cyber insecurity we experience today. Quantum computing does not change the reliance of cryptology on social factors, and in some ways makes it more complicated (Lindsay 2020a).

\section{The Strategic utility of cryptology}

The organizational contest of cryptology is not played for its own sake but in pursuit of more substantive political or economic goals, distributional outcomes, or policy concessions. The political logic of cryptology is implemented by competing organizations in a strategic context of dueling conspiracy. Intelligence collection operations (or criminal theft) is a second-order conspiracy to penetrate a target's first-order conspiracy to gain or preserve the advantages of secrecy. Counterintelligence, likewise, is essentially a third-order conspiracy (i.e., deceiving the deceivers who penetrate deception).

Some of the great success stories in cryptologic history highlight the importance of cryptography for conspiracy and cryptanalysis for defeating it. During her house arrest in 1586, Mary Queen of Scots conspired with a former page to assassinate Elizabeth and foment a Catholic rebellion; they were betrayed by a courier who diverted their enciphered communications to a talented cryptanalyst working for the Crown, and they provided damning evidence for Mary's trial and execution (Kahn 1996: 122-23). A century before the Russian "doxing" of the 
US Democratic National Committee in 2016, the British parlayed a cryptanalytic coup into perhaps the greatest diplomatic influence operation of all time. The single most famous cryptogram in history came from German Secretary for Foreign Affairs Arthur Zimmermann proposing a secret alliance with Mexico against the United States; British interception, decryption, and covert delivery of the telegram to the United States helped President Woodrow Wilson persuade Congress to declare war on Germany (Kahn 1996: 266-97). Both of these were ambitious (if reckless) conspiracies to change the balance of power, and cryptanalysis not only defeated but ultimately spelled disaster for the conspirators. If secrecy was potentially destabilizing, had it succeeded, so was revelation, once it failed.

Cryptography is most useful in situations in which secrecy provides some political advantage for a group and the members of that group have to cooperate to gain that advantage. Specifically, secrecy can provide (1) a political bargaining advantage, (2) a military maneuver advantage, or (3) intellectual property protection.

First, cryptography enables a political group to coordinate bargaining strategy while disguising or exaggerating strengths and weaknesses. Political actors have strategic incentives to misrepresent their power to gain a bargaining advantage (Fearon 1995; Slantchev 2010), and cryptography enables them to do so. A group of negotiators will usually want to rehearse their presentation and conceal their potential concessions and reservation price in order to get the best deal. For example, during the 1921 naval disarmament conference in Washington, Japan was publicly demanding a ratio of ten to seven with the United States and Great Britain. The Japanese Foreign Office cabled its negotiator that it was willing to settle for ten to six but should avoid settling if possible. Unfortunately for the Japanese, the US State Department "Black Chamber" had intercepted and deciphered this cable, which enabled US Secretary of State Charles Hughes to demand, stubbornly and successfully, a ratio of ten to six (Yardley 1931).

Second, the secret coordination of operations covertly shifts costs and benefits. A criminal conspiracy, an espionage ring, and a combined arms military unit all have very different material capabilities, to be sure, but they follow a similar political logic in this respect. These groups rely on maneuver and stealth to evade the defenses of an adversary to concentrate at some decisive point to achieve tactical surprise or steal resources. The local advantage achieved through infiltration and maneuver is fleeting. Members of the group must coordinate their efforts before the adversary can reinforce or counterattack the vulnerable areas that the act of maneuver uncovers. The weaker a group is materially, the less able it is to reinforce its vulnerabilities and the more it depends on stratagem (Kahn 2001).

It is notable that many prominent cryptologic successes occur in military domains such as sea and air that emphasize maneuver and surprise. The legendary exploits of Bletchley Park and the Royal Navy's Operational Intelligence Centre in penetrating German U-boat communications enabled convoy rerouting and antisubmarine targeting in the Battle of the Atlantic (Beesly 2000). The US Navy penetration of Japanese codes provided invaluable insight into the order of battle and fleet movements of the Imperial Japanese Navy and was instrumental 
for victory in the Battles of the Coral Sea and Midway (Parker 2017). Midway is particularly notable as the Japanese intended to fool the Americans with a feint toward the Aleutians and catch them at Midway, but instead the Americans were able to fool the Japanese by appearing at an inopportune moment; the deceivers were deceived (and a deception operation was used to confirm the accuracy of US SIGINT). Cryptography is particularly important in naval warfare, and enemy cryptanalysis particularly dangerous, because the sudden loss of information advantage can be disastrous for expensive "low-density, high-demand" assets. Similarly in air operations, SIGINT can provide valuable targeting data, as Admiral Isoroku Yamamoto found out the hard way in April 1943 after US cryptologists intercepted a report of his travel itinerary (Kahn 1996: 595-601). While land warfare typically depends more on mass and attrition compared to the air and maritime domains, armored warfare relies more on maneuver and surprise, and thus cryptology. German interception of cables from the American military attaché in Cairo reporting on British movements in North Africa "provided Rommel with undoubtedly the broadest and clearest picture of enemy forces and intentions available to any Axis commander throughout the whole war" (Kahn 1996: 473). This, incidentally, is an example of "fourth party" collection, whereby an intelligence service spies on another intelligence service to learn about its targets' communications. The information advantage vanished, however, when the Allies changed attachés and codes, and thus the opening cannonade at Alamein "came as a complete surprise to the Africa Corps" (Kahn 1996: 477).

Third, cryptography protects information resources such as digital money and intellectual property. Information goods are costly to produce and nearly costless to copy, so preventing copying is necessary for creating the scarcity that ensures their value. It is counterintuitive that conspiracies of silence can be stabilizing in this sense. If cryptography can provide advantages in war, it also can be a stabilizing factor in peacetime by protecting the privacy of citizens and legitimate property rights. Most democratic systems of law recognize the sanctity of attorney-client, clerical, and marital privilege, which in effect provide a bargaining advantage to citizens in the courts by protecting their strengths and weaknesses from public scrutiny. Sports teams try to hide their strategies to gain a game time advantage to improve the competitive quality of the game. Some citizens like to protect their personal data from surveillance by advertising firms or cybercriminals. The security of financial transactions and bank accounts from theft and fraud is vital for trust in a system of economic exchange. Cryptography (PKI) provides invaluable protection for all these applications. Legal privacy protections in these examples are provided within an institutional framework that defines property rights and legitimate and illegitimate types of competition. Yet the potential for abuse of legitimate privacy is inherent to the political logic of cryptology. The dilemma of counterintelligence in a democracy is that privacy can both protect citizens and enable traitors (Landau 2010).

Ironically, the availability of strong encryption has contributed to the cyber security epidemic we face today. Robust PKI enables the widespread trust in cyberspace that spies and criminals exploit. For the past two decades, organizations 
and individuals have been hemorrhaging confidential data, and powerful SIGINT agencies have been enjoying a renaissance in technical collection. This is possible in large part because people fail to use encryption properly. Even worse, the availability of strong cryptography can provide a false sense of security for users. The mathematical strength of encryption protocol effectively shifts the incentives for exploitation to other vulnerabilities in the software, hardware, and organizational implementation of the protocol. Gullible humans are the Achilles Heel of classical cryptology, and they will also be the undoing of quantum cryptology.

In all three cases, there is a fundamentally ambiguous relationship between cryptologic advantage and political advantage. Cryptanalytic success can reveal information that makes bargains more likely than conflict. It can also reveal high value targets that make surprise attack more attractive than bargaining. Cryptographic success can deny either of these advantages, which makes stable bargains less and more likely, respectively. Moreover, while the offensive and defensive sides of cryptology are functionally distinct (i.e., cryptanalytic code breaking and cryptographic code making), they do necessarily correlate with political-military offense or defense. A defensively motivated actor can use cryptanalysis to break into an attacker's networks to figure out where to reinforce defenses to blunt a coming attack. An attacker can use cryptography to protect its military communications and preserve the element of surprise which it needs to overwhelm defenders. Cryptographic success can cover cryptanalysis, and cryptanalytic success can prompt cryptographic innovation. These dynamics are fundamentally political in nature, so they are unlikely to be transformed by quantum technology. On the contrary, quantum technology will be enlisted into the service of cryptologic contests.

\section{Quantum possibility and social reality}

The balance between offense and defense in intelligence has always depended more on institutional factors and strategic context than technological architecture. Thus, it will still be possible to collect and protect secrets after the quantum information revolution. In this brief review of the political dynamics of cryptology, we have encountered a broad diversity of actors and applications. The tumultuous relationship between cryptologic technology and political advantage is likely to become even more complex, socially and technically. The overall implications of quantum information technology for strategic stability are profoundly ambiguous, but this ultimately has more to do with the politics of information than the technology of quantum computing.

On the cryptanalytic side (offense), engineers first must overcome formidable challenges such as error correction to build a large-scale universal quantum computer. Quantum computing might then facilitate bulk decryption of intercepted data. It would not, however, improve access and placement to the target's data in the first place, which could still be protected by a smart OPSEC policy. Quantum computing also would not help dramatically with analysis on the back end, although it could help to optimize database searches. It could even complicate 
the analytical process by vastly increasing the number of decrypts that have to be analyzed.

On the cryptographic side (defense), quantum-safe encryption at best offers a restoration to the status quo, temporarily threatened by the advent of quantum computing, rather than a revolution. On the one hand, quantum communication will cancel out whatever problems quantum cryptanalysis creates for communications security. On the other, quantum cryptosystems will still depend on complex sociotechnical implementations that depend, in turn, on lazy, gullible, selfish human beings. Indeed, if quantum computers marginally improve public trust in information networks and thus the data on them, those networks will simply become more attractive intelligence targets.

New levels of trust will create new opportunities for abusing trust. The golden age of cyber espionage today does not need quantum computing to break into organizations that have access to strong cryptosystems. Cyberspace as we know it today already has robust cryptosystems in modern PKI. Future defenses provided by quantum cryptosystems will, similarly, only be as good as the people who use them.

I would like to conclude on a personal note by discussing how I tackled quantum computing as a social scientist (Lindsay 2020a; 2020b). I am fortunate to have a few advantages in this respect, to include training in physics and computer science as an undergraduate and years serving as an intelligence officer in the US Navy prior to pursuing a doctorate in political science. I have written extensively on cyber security and IR, usually from a skeptical perspective (Lindsay 2013; 2014; 2015; 2017; Gartzke and Lindsay 2015; 2017; Lindsay and Gartzke 2017; 2018). Quantum computing caught my attention because I was starting to hear familiar claims about the deterministic potential of new ICT. I immersed myself in technical sources to gain a basic understanding of quantum concepts (Aaronson 2013; Wilde 2017; National Academies of Sciences, Engineering, and Medicine 2019). However, it quickly became apparent that cryptologic history (Kahn 1996; Singh 1999; Alvarez 2000; Aldrich 2010; Parker 2017) would be just as valuable, if not more.

Technology does not determine politics; often it is the other way round. Scholars of cyber security (or quantum computing) must understand the details of information technology, just as scholars of international political economy need to understand the technical nuances of central banking. But they must then go further to interrogate the social context and constitution of these technologies. Technology can at most alter the value of a variable in some theory about some political outcome, such as conflict onset, escalation, duration, or termination. But that variable will almost always be conditioned on other social factors including organizational doctrine, administrative structure, national culture, or elite politics. Through this project, it became apparent to me that the real problem was not that IR lacked an understanding of quantum computing, but that it had little to say about cryptology of any generation. Likewise for cyber security, I have come to believe that the problem is less that IR does not understand information technology and more that IR has only recently started to seriously study intelligence phenomena (Jervis 2011; Rovner 2011; Carson 2018; O’Rourke 2018; Rid 2020). 
The field is wide open for IR to examine the secret side of politics, which increasingly involves the exploitation of information technology. Social scientists can and should reclaim technology from the technologists.

\section{Note}

1 IBM scientists argue that the Oak Ridge Summit could complete the same task in three days rather than 10,000 years, but this does not overturn the basic achievement; future quantum computers using more than Sycamore's 53 qubits will be able to outperform Summit or anything else by a huge margin (Aaronson 2019).

\section{References}

All links checked on August 20, 2021.

Aaronson, S. (2013). Quantum Computing Since Democritus. Cambridge: Cambridge University Press.

Aaronson, S. (2019, October 30). Why Google's Quantum Supremacy Milestone Matters. The New York Times. Retrieved from: https:/www.nytimes.com/2019/10/30/opinion/ google-quantum-computer-sycamore.html

Aldrich, R. (2010). GCHQ: The Uncensored Story of Britain's Most Secret Intelligence Agency. London: HarperCollins.

Alvarez, D. J. (2000). Secret Messages: Codebreaking and American Diplomacy, 19301945. Lawrence, KS: University Press of Kansas.

Arute, F., Arya, K., Babbush, R., Bacon, D., Bardin, J. C., Barends, R., Biswas, R., et al. (2019). Quantum Supremacy Using a Programmable Superconducting Processor. Nature, 574(7779): 505-10.

Beesly, P. (2000). Very Special Intelligence: The Story of the Admiralty's Operational Intelligence Centre, 1939-1945. London: Greenhill Books.

Bernstein, D. J., Heninger, N., Lou, P. and Valenta, L. (2017). Post-Quantum RSA. Cryptology ePrint Archive. Retrieved from: https://eprint.iacr.org/2017/351.pdf.

Biercuk, M. J. and Fontaine, R. (2017, November 17). The Leap into Quantum Technology: A Primer for National Security Professionals. War on the Rocks (blog). Retrieved from: https://warontherocks.com/2017/11/leap-quantum-technology-primer-national-security -professionals/.

Borghard, E. D. and Lonergan, S. W. (2017). The Logic of Coercion in Cyberspace. Security Studies, 26(3): 452-81.

Brantly, A. F. (2016). The Decision to Attack: Military and Intelligence Cyber DecisionMaking. Atlanta, GA: University of Georgia Press.

Brassard, G. (2016). Cryptography in a Quantum World. In: SOFSEM 2016: Theory and Practice of Computer Science, 3-16. Lecture Notes in Computer Science. Berlin, Heidelberg: Springer.

Buchanan, B. (2017). The Cyber Security Dilemma: Hacking, Trust and Fear between Nations. New York: Oxford University Press.

Buchmann, J., Lauter, K., and Mosca, M. (2017). Postquantum Cryptography - State of the Art. IEEE Security Privacy, 15(4): 12-13. https://doi.org/10.1109/MSP.2017 .3151326 .

Carson, A. (2018). Secret Wars: Covert Conflict in International Politics. Princeton: Princeton University Press. 
Chen, L., Jordan, S., Liu, Y.-K., Moody, D., Peralta, R., Perlner, R., and Smith-Tone, D. (2016). Report on Post-Quantum Cryptography. NISTIR 8105. Gaithersburg, MD: National Institute of Standards and Technology.

Copeland, B. J. (ed.). (2010). Colossus: The Secrets of Bletchley Park's Code-Breaking Computers. New York: Oxford University Press.

Deibert, R. J. (2013). Black Code: Inside the Battle for Cyberspace. Toronto, ON: McClelland \& Stewart.

DigiCert (2018). Check Our Numbers: The Math Behind Estimations to Break a 2048-Bit Certificate. Archive copy. Retrieved from: https://web.archive.org/web /20181004033325/https://www.digicert.com/TimeTravel/math.htm.

Dowling, J. P. and Milburn, G. J. (2003). Quantum Technology: The Second Quantum Revolution. Philosophical Transactions. Series A, Mathematical, Physical, and Engineering Sciences, 361(1809): 1655-74.

Dunn Cavelty, M. (2008). Cyber-Terror - Looming Threat or Phantom Menace? The Framing of the US Cyber-Threat Debate. Journal of Information Technology \& Politics, 4(1): 19-36.

Fearon, J. D. (1995). Rationalist Explanations for War. International Organization, 49(3): 379-414.

Feynman, R. P. (1982). Simulating Physics with Computers. International Journal of Theoretical Physics, 21(6/7): 467-88.

Gartzke, E. (2013). The Myth of Cyberwar: Bringing War in Cyberspace Back Down to Earth. International Security, 38(2): 41-73.

Gartzke, E. and Lindsay, J. R. (2015). Weaving Tangled Webs: Offense, Defense, and Deception in Cyberspace. Security Studies, 24(2): 316-48.

Gartzke, E. and Lindsay, J. R. (2017). Thermonuclear Cyberwar. Journal of Cyber security, 3(1): 37-48.

Gidney, C. and Ekera, M. (2019). How to Factor 2048 Bit RSA Integers in 8 Hours Using 20 Million Noisy Qubits. arXiv. Retrieved from: https://arxiv.org/pdf/1905.09749.pdf.

Grey, C. (2012). Decoding Organization: Bletchley Park, Codebreaking and Organization Studies. New York: Cambridge University Press.

Hurd, W. (2017, December 7). Quantum Computing Is the Next Big Security Risk. Wired. Retrieved from: https://www.wired.com/story/quantum-computing-is-the-next-big -security-risk/.

Jervis, R. (2011). Why Intelligence Fails: Lessons from the Iranian Revolution and the Iraq War. Ithaca, NY: Cornell University Press.

Kahn, D. (1996). The Codebreakers: The Comprehensive History of Secret Communication from Ancient Times to the Internet. Revised. New York: Simon and Schuster.

Kahn, D. (2001). An Historical Theory of Intelligence. Intelligence and National Security, 16(3): 79-92.

Kania, E. B. (2018, September 28). China's Quantum Future. Foreign Affairs. Retrieved from: https://www.foreignaffairs.com/articles/china/2018-09-26/chinas-quantum-future.

Kania, E. B. and Costello, J. (2018). Quantum Hegemony? China's Ambitions and the Challenge to U.S. Innovation Leadership. Washington, DC: Center for a New American Security.

Kello, L. (2013). The Meaning of the Cyber Revolution: Perils to Theory and Statecraft. International Security, 38(2): 7-40.

Kenny, R. (2017, November 28). The Quantum Gap with China. Foreign Policy. Retrieved from: https://foreignpolicy.com/2017/11/28/the-quantum-gap-with-china/. 
Kreps, S. and Schneider, J. (2019). Escalation Firebreaks in the Cyber, Conventional, and Nuclear Domains: Moving beyond Effects-Based Logics. Journal of Cyber security, 5(1). https://doi.org/10.1093/cybsec/tyz007.

Landau, S. E. (2010). Surveillance or Security? The Risks Posed by New Wiretapping Technologies. Cambridge, MA: MIT Press.

Lawson, S. (2013). Beyond Cyber-Doom: Assessing the Limits of Hypothetical Scenarios in the Framing of Cyber-Threats. Journal of Information Technology \& Politics, 10(1): $86-103$.

Libicki, M. C. (2007). Conquest in Cyberspace: National Security and Information Warfare. New York: Cambridge University Press.

Liff, A. P. (2012). Cyberwar: A New 'Absolute Weapon'? The Proliferation of Cyberwarfare Capabilities and Interstate War. The Journal of Strategic Studies, 35(3): 401-28.

Lindsay, J. R. (2013). Stuxnet and the Limits of Cyber Warfare. Security Studies, 22(3): 365-404.

Lindsay, J. R. (2014). The Impact of China on Cyber Security: Fiction and Friction. International Security, 39(3): 7-47.

Lindsay, J. R. (2015). Tipping the Scales: The Attribution Problem and the Feasibility of Deterrence against Cyber Attack. Journal of Cyber security, 1(1): 53-67.

Lindsay, J. R. (2020a). Demystifying the Quantum Threat: Infrastructure, Implementation, and Intelligence Advantage. Security Studies, 29(2): 335-361.

Lindsay, J. R. (2020b). Surviving the Quantum Cryptocalypse. Strategic Studies Quarterly, 14(2): 49-73.

Lindsay, J. R. and Gartzke, E. (2017). Cross-Domain Deterrence and Cyber Security: The Consequences of Complexity. In: D. van Puyvelde and A. F. Brantley (eds), US National Cyber Security: International Politics, Concepts and Organization. New York: Routledge, pp. 11-27.

Lindsay, J. R. and Gartzke, E. (2018). Coercion through Cyberspace: The Stability-Instability Paradox Revisited. In: K. M. Greenhill and P. Krause (eds), Coercion: The Power to Hurt in International Politics. New York: Oxford University Press, pp. 179-203.

Lo, H.-K. and Lütkenhaus, N. (2007). Quantum Cryptography: From Theory to Practice. Physics in Canada, 63(4): 191-96.

Lynn III, W. J. (2010). Defending a New Domain: The Pentagon's Cyberstrategy. Foreign Affairs, 89(5): 97-108.

Mulholland, J., Mosca, M. and Braun, J. (2017). The Day the Cryptography Dies. IEEE Security Privacy, 15(4): 14-21.

National Academies of Sciences, Engineering, and Medicine (2019). Quantum Computing: Progress and Prospects. Washington, DC: The National Academies Press.

Nikias, C. and Max, L. (2018, May 11). This Is the Most Important Tech Contest since the Space Race, and America Is Losing. Washington Post. Retrieved from: https://www .washingtonpost.com/opinions/this-is-the-most-important-tech-contest-since-the-space -race-and-america-is-losing/2018/05/11/7a4a4772-4e21-11e8-b725-92c89fe3ca4c story.html

O'Rourke, L. A. (2018). Covert Regime Change: America's Secret Cold War. Ithaca, NY: Cornell University Press.

Owen, T. and Gorwa, R. (2016, September 7). Quantum Leap: China's Satellite and the New Arms Race. Foreign Affairs. Retrieved from: https://www.foreignaffairs.com/ articles/2016-09-07/quantum-leap 
Parker, F. D. (2017). A Priceless Advantage: U.S. Navy Communications Intelligence and the Battles of Coral Sea, Midway, and the Aleutians. 3rd ed. Vol. 5. United States Cryptologic History, World War II, IV. Fort George G. Meade, MD: Center for Cryptologic History, National Security Agency.

Putnam, R. D. (1988). Diplomacy and Domestic Politics: The Logic of Two-Level Games. International Organization, 42(3): 427-60.

Ratcliff, R. A. (2006). Delusions of Intelligence: Enigma, Ultra, and the End of Secure Ciphers. New York: Cambridge University Press.

Rattray, G. J. (2001). Strategic Warfare in Cyberspace. Cambridge, MA: MIT Press.

Rid, T. (2012). "Cyber War Will Not Take Place“. The Journal of Strategic Studies, 35(5): 5-32.

Rid, T. (2020). Active Measures: The Secret History of Disinformation and Political Warfare. New York: Farrar, Straus and Giroux.

Rid, T. and Buchanan, B. (2015). Attributing Cyber Attacks. Journal of Strategic Studies, 38(1-2): 4-37.

Rovner, J. (2011). Fixing the Facts: National Security and the Politics of Intelligence. Ithaca, NY: Cornell University Press.

Schneider, J. (2019). The Capability/Vulnerability Paradox and Military Revolutions: Implications for Computing, Cyber, and the Onset of War. Journal of Strategic Studies, 42(6): 841-63. https://doi.org/10.1080/01402390.2019.1627209.

Shannon, C. E. (1945). A Mathematical Theory of Cryptography. Technical Report MM 45-110-02. Holmdel: Bell Labs.

Shannon, C. E. (1948). A Mathematical Theory of Communication. The Bell System Technical Journal, 27(October): 379-423, 623-656.

Shannon, C. E. (1951). The Redundancy of English. In: Transactions of the 7th Conference on Cybernetics. New York: Josiah Macy, Jr. Foundation, pp. 248-272.

Simon, C. (2017). Towards a Global Quantum Network. Nature Photonics, 11(11): 67880. https://doi.org/10.1038/s41566-017-0032-0.

Singh, S. (1999). The Code Book: The Science of Secrecy from Ancient Egypt to Quantum Cryptography. New York: Random House.

Slantchev, B. L. (2010). Feigning Weakness. International Organization, 64(3): 357-88.

Slayton, R. (2017). What Is the Cyber Offense-Defense Balance? Conceptions, Causes, and Assessment. International Security, 41(3): 72-109.

Smeets, M. (2017). A Matter of Time: On the Transitory Nature of Cyberweapons. Journal of Strategic Studies, 41(1-2): 6-32.

Thirsk, J. W. (2001). Traffic Analysis: A Log-Reader's Tale. In: M. Smith and R. Erskine (eds), Action This Day: Bletchley Park from the Breaking of the Enigma Code to the Birth of the Modern Computer. New York: Bantam Press, pp. 264-77.

Valeriano, B. and Maness, R. C. (2015). Cyber War versus Cyber Realities: Cyber Conflict in the International System. New York: Oxford University Press.

Warner, M. and Benson, R. L. (1997). Venona and Beyond: Thoughts on Work Undone. Intelligence and National Security, 12(3): 1-13.

Wehner, S., Elkouss, D. and Hanson, R. (2018). Quantum Internet: A Vision for the Road Ahead. Science, 362(6412): eaam9288.

Wilde, M. M. (2017). Quantum Information Theory. 2nd ed. New York: Cambridge University Press.

Yardley, H. O. (1931). The American Black Chamber. Indianapolis, IN: Bobbs-Merrill. 